\title{
Carotid Plaque Score and Risk of Cardiovascular Mortality in the Oldest Old: Results from the TOOTH Study
}

\author{
Takumi Hirata ${ }^{1}$, Yasumichi Arai ${ }^{1}$, Michiyo Takayama ${ }^{2}$, Yukiko Abe ${ }^{1}$, Kiyoshi Ohkuma ${ }^{3}$ and \\ Toru Takebayashi ${ }^{4}$ \\ ${ }^{1}$ Center for Supercentenarian Medical Research, Keio University School of Medicine, Tokyo, Japan \\ ${ }^{2}$ Center for Preventive Medicine, Keio University School of Medicine, Tokyo, Japan \\ ${ }^{3}$ Department of Radiology, Keio University School of Medicine, Tokyo, Japan \\ ${ }^{4}$ Department of Preventative Medicine and Public Health, Keio University School of Medicine, Tokyo, Japan
}

Aim: Accumulating evidence suggests that predictability of traditional cardiovascular risk factors declines with advancing age. We investigated whether carotid plaque scores (CPSs) were associated with cardiovascular disease (CVD) death in the oldest old, and whether asymmetrical dimethylarginine (ADMA), a marker of endothelial dysfunction, moderated the association between the CPS and CVD death.

Methods: We conducted a prospective cohort study of Japanese subjects aged $\geq 85$ years without CVD at baseline. We followed this cohort for 6 years to investigate the association of CPS with CVD death via multivariable Cox proportional hazard analysis. We divided participants into three groups according to CPS (no, 0 points; low, $1.2-4.9$ points; high, $\geq 5.0$ points). The predictive value of CPS for estimating CVD death risk over CVD risk factors, including ADMA, was examined using C-statistics. Results: We analyzed 347 participants (151 men, 196 women; mean age, 87.6 years), of which 135 $(38.9 \%)$ had no carotid plaque at baseline, and $48(13.8 \%)$ had high CPS. Of the total, $29(8.4 \%)$ participants experienced CVD-related death during the study period. Multivariable analysis revealed a significant association of high CPS with CVD-related mortality relative to no CPS (hazard ratio, 3.90; 95\% confidence interval: 1.47-10.39). ADMA was not associated with CVD death, but the significant association between CPS and CVD death was observed only in lower ADMA level. The addition of CPS to other risk factors improved the predictability of CVD death $(p=0.032)$.

Conclusions: High CPS correlated significantly with a higher CVD death risk in the oldest old with low cardiovascular risk. Ultrasound carotid plaque evaluation might facilitate risk evaluations of CVD death in the very old.

Key words: Carotid plaque score, Cardiovascular disease, Asymmetrical dimethylarginine, Oldest old, Epidemiology

\section{Introduction}

Cardiovascular disease (CVD)-related mortality has increased exponentially with increasing age worldwide $^{1-3)}$. Atherosclerosis is the major cause of CVD, and the appropriate management of several related

Address for correspondence: Yasumichi Arai, Center for Supercentenarian Medical Research, Keio University School of Medicine, 35 Shinanomachi, Shinjuku-ku, Tokyo, 160-8582, Japan

E-mail: yasumich@keio.jp

Received: August 29, 2016

Accepted for publication: December 12, 2016 risk factors is an essential step toward CVD prevention. Current guidelines for the primary prevention of CVD recommend that initial risk assessments to identify adults at high risk for CVD should be based on traditional atherosclerosis risk factors ${ }^{4}$. Consequently, various tools used for CVD risk assessments are widely used in the general population, including the Framingham risk score ${ }^{5)}$ and the QRISK2 ${ }^{6}$. However, the predictive power of CVD risk factors reportedly decreases with advancing age ${ }^{7-9)}$.

Bilateral carotid echography has recently been used to evaluate the presence of subclinical atherosclerosis. The presence of carotid plaques, as measured by 
echography, has been shown to serve as a surrogate marker of subclinical atherosclerosis. Several reports have also shown associations of carotid plaques with coronary heart disease and carotid intima-media thickness (IMT) ${ }^{10,11)}$. Another cohort study reported that CVD event risk prediction could be improved by adding carotid plaque metrics to traditional Framingham risk factors in a multi-ethnic population aged 45-84 years without $\mathrm{CVD}^{12}$. In addition, the presence of carotid plaques, but not carotid IMT, was shown to be an independent predictor of coronary heart disease in adults aged $65-85$ years $^{13)}$. These studies suggest that the presence of carotid plaques remains a useful predictor of CVD risk in adults younger than 85 years. However, no studies have reported the relationship between the carotid plaque score (CPS) and the incidence of CVD in a general population aged $\geq 85$ years.

Additionally, asymmetrical dimethylarginine (ADMA), an endogenous inhibitor of nitric oxide (NO) synthase, is a novel biomarker of endothelial dysfunction, and high levels of this molecule have been associated with incident CVD and mortality in patients with coronary artery disease, renal failure, and diabetes mellitus ${ }^{14-17)}$. Plasma ADMA levels increase with age in the Framingham Offspring Study ${ }^{18)}$, and ADMA may be involved in atherogenic processes relevant to aging, such as endothelial cell senescence ${ }^{19)}$. Based on these findings, we hypothesized that ADMA is a possible moderator for the association between atherosclerosis and CVD death in the very old.

\section{Aim}

We aimed to clarify 1) the association between the CPS and cardiovascular mortality in a general Japanese population aged $\geq 85$ years without clinical CVD and 2) whether ADMA moderated the association between the CPS and cardiovascular mortality.

\section{Methods}

\section{Design, Setting, and Participants}

We used data from the Tokyo Oldest Old Survey on Total Health (TOOTH) study. The TOOTH study, a community-based prospective cohort study, was established in 2008 to study the physical, mental, and oral health of the oldest old individuals living in the Tokyo Metropolitan area, Japan. Details of the TOOTH study have been described elsewhere ${ }^{20)}$. The TOOTH study was previously registered in the University Hospital Medical Information Network Clinical Trial Registry (ID: UMIN000001842). In addition, the present study was approved by the ethics committee of the Keio University School of Medicine (approval number: 20070047). Written informed consent was obtained from all participants.

In the present study, a total of 542 Japanese participants ( 236 men, 306 women) aged $\geq 85$ years participated in the baseline survey from March 2008 to November 2009. We excluded 120 participants who were diagnosed with CVD at baseline, 61 who had not undergone carotid echography, and 14 with missing data. We finally analyzed data for 347 participants (151 men, 196 women; mean age, 87.6 years) without CVD. We stratified participants according to CPS into no (0 points), low (1.2-4.9 points), and high ( $\geq 5.0$ points) CPS groups.

\section{Baseline Examination}

All participants were assessed by trained geriatricians to determine their previous medical histories, medication use, and lifestyle risk factors (including smoking and alcohol drinking). The following diagnostic criteria were used for medical conditions. Hypertension was defined as a systolic blood pressure (BP) $\geq 140 \mathrm{mmHg}$, diastolic $\mathrm{BP} \geq 90 \mathrm{mmHg}$, or current use of medication for hypertension. Dyslipidemia was defined as a total cholesterol (TC) level $\geq 5.70$ $\mathrm{mmol} / \mathrm{L}$, high-density lipoprotein cholesterol (HDLC) level $<1.04 \mathrm{mmol} / \mathrm{L}$, or current use of medication for dyslipidemia. Diabetes was defined as a non-fasting glucose level $\geq 11.1 \mathrm{mmol} / \mathrm{L}$, glycated hemoglobin (HbA1c) level $\geq 6.5 \%$, or current use of medication for diabetes.

Height and body weight were measured by trained staff while participants wore socks and light clothing. Body mass index (BMI) was calculated by dividing the weight in kilograms by the squared height in meters. While the participants were seated, BP was measured twice using an automatic sphygmomanometer after a minimum 5-min rest interval. We used the average of the two measurements for the analysis. Pulse pressure (PP) was defined as the difference between systolic BP and diastolic BP.

Non-fasting blood samples were collected from all participants and transported to a clinical laboratory (SRL Inc., Tokyo, Japan) for analysis. We measured serum TC, triglyceride (TG), and HDL-C levels using standard enzymatic methods. Low-density lipoprotein cholesterol (LDL-C) was calculated using Friedwald's formula when the TG level was $<400 \mathrm{mg} / \mathrm{dL}$, and the serum non-HDL-C level was calculated by subtracting the HDL-C level from the TC level. The HbA1c level was determined using high-performance liquid chromatography and reported according to the guidelines of the National Glycohemoglobin Standardization Program. The serum C-reactive protein (CRP) level was measured using a latex turbidimetric immunoas- 
Table 1. Characteristics of participants at baseline according to the carotid plaque score

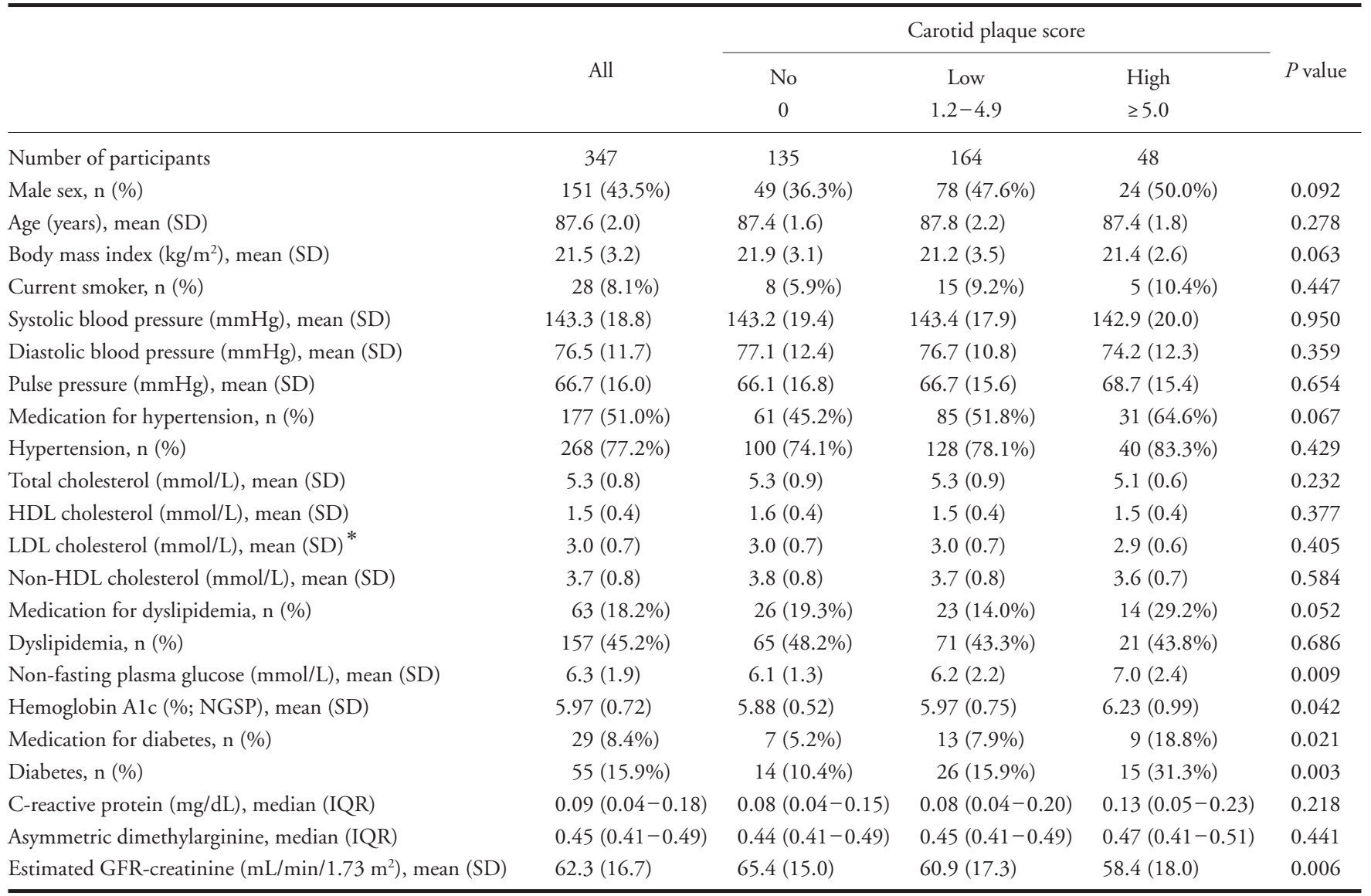

${ }^{*} n=345$

Abbreviations: $\mathrm{HDL}=$ high density lipoprotein, $\mathrm{LDL}=$ low density lipoprotein, $\mathrm{GFR}=$ glomerular filtration rate, $\mathrm{SD}=$ standard deviation, $\mathrm{IQR}=$ interquartile range

say, and the serum ADMA level was determined via high-performance liquid chromatography. The intraassay and inter-assay coefficients of variation were $2.97 \%$ and $2.50 \%$, respectively. The serum creatinine level was measured using a standard enzymatic method, and the estimated glomerular filtration rate (eGFR) was calculated using the following formula: eGFR $(\mathrm{mL} /$ $\left.\mathrm{min} / 1.73 \mathrm{~m}^{2}\right)=194^{*}[\text { serum creatinine }(\mathrm{mg} / \mathrm{dL})]^{-1.094 *}$ [age (years) $]^{-0.287}\left[{ }^{*} 0.739 \text { (if female) }\right]^{21)}$.

Two-dimensional ultrasound examinations of the bilateral common carotid arteries were performed using a $10-\mathrm{MHz}$ linear transducer (Hitachi EUB-525, Hitachi Medical, Tokyo, Japan). A trained physician blinded to the participants' clinical information performed all ultrasound examinations at the clinical site. Of the total, 61 participants who were examined at home were not eligible for this procedure. Definitions of the carotid segments were adopted from the Cardiovascular Health Study ${ }^{22}$. An anterolateral approach was used to obtain a longitudinal image of the com- mon carotid artery, and we determined the presence of focal plaque and/or calcific deposits through a thorough search of the common and internal carotid arteries. Carotid plaque was defined as a clearly identified area of focally increased thickness in the intima-media layer $(>1.2 \mathrm{~mm})$, rather than using an IMT cut-off value as previously described ${ }^{23)}$. CPS was calculated by summing the thickness of all plaques in the bilateral common and internal carotid arteries.

\section{Follow-Up Survey and Outcome Measurements}

All participants were prospectively followed up for 6 years (till December 2015) via annual telephone contact or mail survey. The primary outcome was CVD death, which included any ischemic heart disease (defined using ICD-10 codes I20-I25), heart failure (I50.0), diseases of arteries (I70-I79) or stroke (defined using ICD-10 codes I60-I63, I69.0-I69.3, or G45). Events were determined based on information provided by the participants' family or by caregiv- 


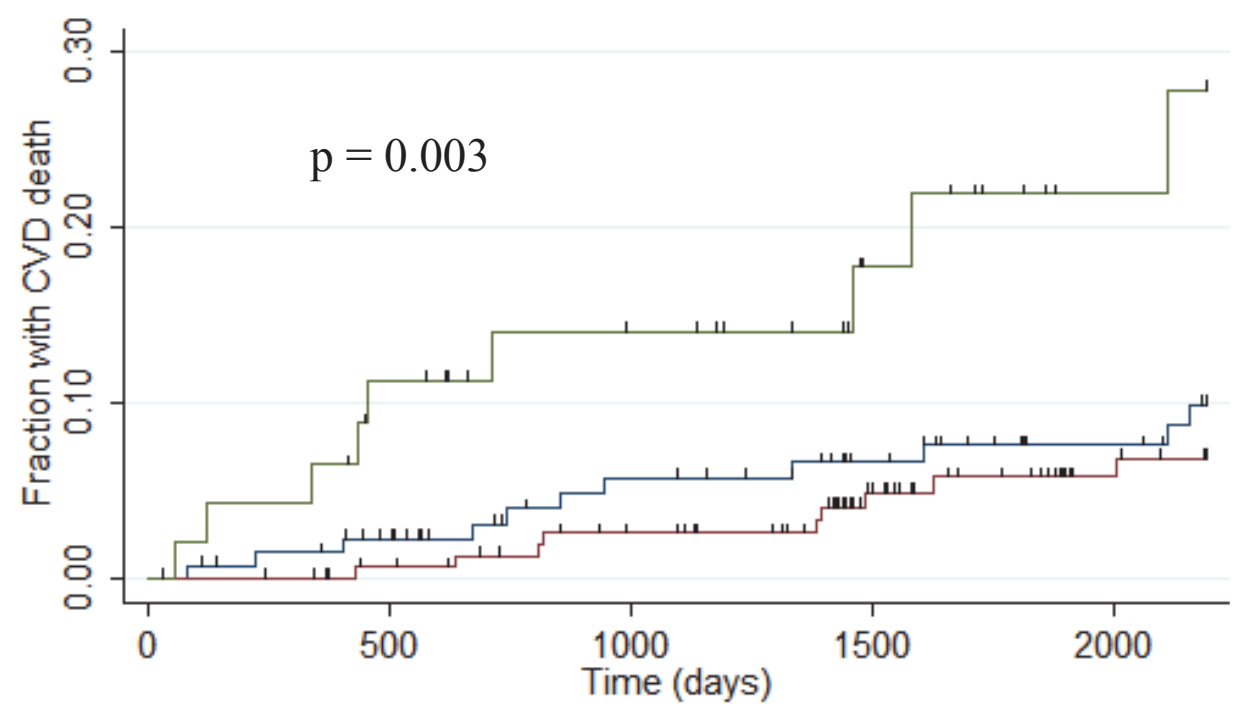

Number at risk

\begin{tabular}{rccccc|} 
No plaque & 135 & 126 & 113 & 100 & 90 \\
Low PS & 164 & 157 & 147 & 118 & 96 \\
High PS & 48 & 40 & 34 & 24 & 17 \\
\cline { 2 - 5 } & & No plaque & & Low plaque score \\
\cline { 3 - 5 } & High plaque score & &
\end{tabular}

Fig. 1. Kaplan-Meier curves of cardiovascular disease mortality according to the carotid plaque score (PS; $n=334$ ). Patients were divided into three groups according to carotid PS (no, 0 points; low, $1.2-4.9$ points; high, $\geq 5.0$ points). $P$ values were calculated using log-rank tests.

ers via annual telephone contact or mail survey. When a participant's death was reported, a geriatrician called the family or a proxy to inquire about the date, place, and cause of death. Follow-up was continued until one of the following censoring events occurred: death from any cause, loss to follow-up (e.g., moving away from the Tokyo Metropolitan area), or the end of the 6-year follow-up period.

\section{Statistical Analysis}

Continuous variables are shown as means and standard deviations or medians and interquartile ranges, and categorical variables are shown as numbers and proportions of participants. We compared the characteristics of participants according to CPS groups using an unpaired $t$-test or the Mann-Whitney $U$ test for continuous variables, and the chi-square test or Fisher's exact test for categorical variables.

We estimated the cumulative cardiovascular mortality in each group using Kaplan-Meier survival curves and compared differences among the three CPS groups using the log-rank test. We used a Cox proportional hazard model to estimate the hazard ratios (HRs) with corresponding 95\% confidence intervals (95\% CIs) after adjusting for potential confounding factors; the low CPS group was used as a reference in all respective analyses. The confounding factors were sex (categorical variable: male, female), age (continuous variable), current smoker (categorical variable: yes, no), BMI (continuous variable), systolic BP (continuous variable) or $\mathrm{PP}$ (continuous variable), TC (continuous variable), HDL-C (continuous variable), HbA1c (continuous variable), estimated GFR (continuous variable), CRP (continuous variable; log-transformed), and ADMA (continuous variable; log-transformed). In addition, we performed sensitivity analysis considering competing risk of death from any causes other than CVD. Multivariate Fine and Gray's semiparametric proportional subdistribution hazards models were used to show sub-hazard ratios (SHRs) and 95\% CIs for CVD death and non-CVD death in a competing risk situation ${ }^{24)}$. We also performed the same analyses stratified by ADMA level $(<0.46 \mu \mathrm{mol} / \mathrm{L}$ or $\geq 0.46$ $\mu \mathrm{mol} / \mathrm{L}$; divided by median).

Finally, we examined the abilities of two models to predict 6-year CVD death using receiver operating characteristic (ROC) curve analyses. The first (basic) model included traditional risk factors such as sex, age, smoking status, BMI, systolic BP, TC, HDL-C, HbA1c, estimated GFR, CRP, and ADMA level. The 
Table 2. Results of a multivariable Cox proportional hazard model with regard to CVD death

\begin{tabular}{|c|c|c|c|c|c|c|c|c|c|}
\hline & \multicolumn{3}{|c|}{ Univariate } & \multicolumn{6}{|c|}{ Multivariate } \\
\hline Male sex & 0.81 & $0.38-1.72$ & 0.591 & 0.82 & $0.35-1.90$ & 0.642 & 0.81 & $0.35-1.88$ & 0.629 \\
\hline Age, per 1 years & 1.20 & $1.06-1.36$ & 0.004 & 1.26 & $1.09-1.46$ & 0.002 & 1.27 & $1.09-1.47$ & 0.002 \\
\hline Systolic blood pressure, per $10 \mathrm{mmHg}$ & 0.91 & $0.75-1.11$ & 0.359 & 0.93 & $0.76-1.14$ & 0.514 & & & \\
\hline Pulse pressure, per $10 \mathrm{mmHg}$ & 0.95 & $0.76-1.20$ & 0.692 & & & & 0.98 & $0.78-1.25$ & 0.896 \\
\hline Total cholesterol, per $1 \mathrm{mmol} / \mathrm{L}$ & 1.22 & $0.80-1.86$ & 0.367 & 1.67 & $1.04-2.70$ & 0.036 & 1.65 & $1.02-2.67$ & 0.040 \\
\hline HDL cholesterol, per $1 \mathrm{mmol} / \mathrm{L}$ & 1.52 & $0.62-3.69$ & 0.358 & 1.13 & $0.39-3.24$ & 0.819 & 1.10 & $0.38-3.13$ & 0.862 \\
\hline Hemoglobin A1c, per $1 \%$ & 0.99 & $0.59-1.66$ & 0.976 & 0.96 & $0.54-1.70$ & 0.893 & 0.96 & $0.54-1.70$ & 0.893 \\
\hline \multicolumn{10}{|l|}{ Carotid plaque score } \\
\hline No (0 points) & Ref. & & & Ref. & & & Ref. & & \\
\hline Low (1.2-4.9 points) & 0.67 & $0.28-1.62$ & 0.377 & 0.58 & $0.23-1.46$ & 0.248 & 0.58 & $0.23-1.46$ & 0.245 \\
\hline High ( $\geq 5.0$ points) & 2.90 & $1.20-7.01$ & 0.018 & 3.90 & $1.47-10.39$ & 0.006 & 3.84 & $1.45-10.20$ & 0.007 \\
\hline
\end{tabular}

$n=347,{ }^{*} \log$-transformed

Multivariate adjustment: Model 1 adjusted for sex, age, body mass index, smoking status, systolic blood pressure, total cholesterol, HDL cholesterol, hemoglobin A1c, C-reactive protein (log-transformed), asymmetric dimethylarginine (log-transformed), and estimated GFR. Model 2 adjusted for sex, age, body mass index, smoking status, pulse pressure, total cholesterol, HDL cholesterol, hemoglobin A1c, C-reactive protein (logtransformed), asymmetric dimethylarginine (log-transformed), and estimated GFR.

Abbreviations: $\mathrm{CVD}=$ cardiovascular disease, $\mathrm{HDL}=$ high density lipoprotein, $\mathrm{GFR}=$ glomerular filtration rate, $\mathrm{HR}=\mathrm{hazard}$ ratio, $\mathrm{CI}=\mathrm{confidence}$ interval

second model included all factors from the basic model, as well as the CPS groups. We calculated Harrell's $\mathrm{C}$ statistics to compare the two models.

All analyses were performed using STATA SE 13 data analysis and statistical software (Stata Corp LP, College Station, TX, USA). All $P$ values for the statistical tests were two-tailed, and a $P$ value $<0.05$ was considered statistically significant.

\section{Results}

We analyzed data of 347 participants (151 men, 196 women; mean age, 87.6 years) without a history of CVD. All participants were included in the estimations of cumulative cardiovascular mortality and HRs for 6-year CVD death. During a follow-up comprising 609,898 person-years over a 6-year period, 109 participants $(31.4 \%)$ died, and $29(8.4 \%)$ died from CVD-related causes (ischemic heart disease death, 13; heart failure death, 4; rupture of thoracic aortic aneurysm, 1 ; ischemic stroke death, 4; cerebral hemorrhage death, 2; subarachnoid hemorrhage death, 1 ; and sudden death, 4).

Table 1 presents the baseline clinical characteris- tics of the participants according to CPS categories. The following results were notable: the mean BMI was $21.5 \mathrm{~kg} / \mathrm{m}^{2}$; only $8.1 \%$ of participants were current smokers; the mean systolic BP was $143.3 \mathrm{mmHg}$, and $51.0 \%$ of participants took medication for hypertension; the mean TC was $5.3 \mathrm{mmol} / \mathrm{L}$, and $18.2 \%$ of participants took medication for dyslipidemia; and the mean $\mathrm{HbA} 1 \mathrm{c}$ was $5.97 \%$, and only $8.4 \%$ of participants took medication for diabetes. Of the total, 212 participants $(61.1 \%)$ had carotid plaques, and 48 $(13.8 \%)$ had a high CPS. The proportion of participants with diabetes was significantly higher, and the estimated GFR was significantly lower among participants with a high CPS versus those with no CPS. In addition, the non-fasting plasma glucose and $\mathrm{HbA1c}$ levels were significantly higher among participants with a high CPS. However, CPS was not found to associate with the CRP or ADMA level.

Cumulative cardiovascular mortality according to CPS stratification is shown in Fig. 1. The model based on CPS categories indicated significant differences among the groups, wherein participants with a high CPS had a higher likelihood of CVD mortality than those with no or low CPS ( $p=0.003$ by the log-rank 
Table 3. Results of a multivariable Cox proportional hazard model with regard to CVD death considering competing risk of death from any causes other than CVD

\begin{tabular}{|c|c|c|c|c|c|c|}
\hline & \multicolumn{3}{|c|}{ Model 1} & \multicolumn{3}{|c|}{ Model 2} \\
\hline Age, per 1 years & 1.27 & $1.08-1.49$ & 0.004 & 1.27 & $1.08-1.51$ & 0.004 \\
\hline Body mass index, per $1 \mathrm{~kg} / \mathrm{m}^{2}$ & 0.91 & $0.79-1.04$ & 0.168 & 0.90 & $0.78-1.04$ & 0.142 \\
\hline Current smoker, yes & 0.51 & $0.07-3.67$ & 0.505 & 0.49 & $0.07-3.62$ & 0.485 \\
\hline Pulse pressure, per $10 \mathrm{mmHg}$ & & & & 1.02 & $0.81-1.29$ & 0.872 \\
\hline Total cholesterol, per $1 \mathrm{mmol} / \mathrm{L}$ & 1.70 & $0.98-2.97$ & 0.060 & 1.69 & $0.97-2.97$ & 0.065 \\
\hline HDL cholesterol, per $1 \mathrm{mmol} / \mathrm{L}$ & 1.03 & $0.37-2.85$ & 0.959 & 1.00 & $0.36-2.75$ & 0.997 \\
\hline Hemoglobin A1c, per $1 \%$ & 0.93 & $0.54-1.60$ & 0.792 & 0.93 & $0.54-1.62$ & 0.801 \\
\hline C-reactive protein, per $1 \mathrm{mg} / \mathrm{dL}^{*}$ & 1.17 & $0.79-1.72$ & 0.439 & 1.17 & $0.79-1.74$ & 0.428 \\
\hline Low (1.2-4.9 points) & 0.59 & $0.24-1.47$ & 0.258 & 0.59 & $0.24-1.46$ & 0.252 \\
\hline High ( $\geq 5.0$ points) & 3.14 & $1.06-9.30$ & 0.039 & 3.09 & $1.06-9.05$ & 0.039 \\
\hline
\end{tabular}

$n=347,{ }^{*} \log$-transformed

Multivariate adjustment: Model 1 adjusted for sex, age, body mass index, smoking status, systolic blood pressure, total cholesterol, HDL cholesterol, hemoglobin A1c, C-reactive protein (log-transformed), asymmetric dimethylarginine (log-transformed), and estimated GFR. Model 2 adjusted for sex, age, body mass index, smoking status, pulse pressure, total cholesterol, HDL cholesterol, hemoglobin A1c, C-reactive protein (logtransformed), asymmetric dimethylarginine (log-transformed), and estimated GFR.

Abbreviations: $\mathrm{CVD}=$ cardiovascular disease, $\mathrm{HDL}=$ high density lipoprotein, $\mathrm{GFR}=$ glomerular filtration rate, SHR=sub-hazard ratio, $\mathrm{CI}=$ confidence interval

Table 4. Multivariate adjusted hazard ratios for carotid plaque score with CVD death considering competing risk of death from any causes other than CVD stratified by ADMA level

\begin{tabular}{|c|c|c|c|c|c|c|}
\hline & \multicolumn{3}{|c|}{$\begin{array}{l}\text { Lower ADMA level } \\
(<0.46 \mu \mathrm{mol} / \mathrm{L})\end{array}$} & \multicolumn{3}{|c|}{$\begin{array}{l}\text { Higher ADMA level } \\
\qquad(\geq 0.46 \mu \mathrm{mol} / \mathrm{L})\end{array}$} \\
\hline & SHR & $95 \% \mathrm{CI}$ & $P$ value & SHR & $95 \% \mathrm{CI}$ & $P$ value \\
\hline \multicolumn{7}{|l|}{ Carotid plaque score } \\
\hline No (0 points) & Ref. & & & Ref. & & \\
\hline Low (1.2-4.9 points) & 0.42 & $0.11-1.56$ & 0.195 & 0.63 & $0.12-3.13$ & 0.567 \\
\hline High ( $\geq 5.0$ points) & 5.51 & $1.45-20.92$ & 0.012 & 1.66 & $0.29-9.32$ & 0.568 \\
\hline
\end{tabular}

$n=347,{ }^{*} \log$-transformed

Multivariate adjustment: Model adjusted for sex, age, body mass index, smoking status, systolic blood pressure, total cholesterol, HDL cholesterol, hemoglobin A1c, C-reactive protein (log-transformed), and estimated GFR.

Abbreviations: $\mathrm{CVD}=$ cardiovascular disease, $\mathrm{HDL}=$ high density lipoprotein, $\mathrm{GFR}=$ glomerular filtration rate, SHR=sub-hazard ratio, $\mathrm{CI}=$ confidence interval, $\mathrm{ADMA}=$ asymmetric dimethylarginine

test). In addition, Table 2 shows the association between CPS and CVD death in the multivariate analysis. The HR for the high CPS group was significantly higher than that for the no CPS group after adjusting for potential confounders (Table 2; HR, 3.90; 95\% CI, 1.47-10.39). In this analysis, neither the ADMA nor CRP level was associated with cardio- vascular mortality. Age and TC level were both significantly associated with CVD death in the multivariate analysis; however, other traditional risk factors, including the systolic BP and HbA1c levels, were not associated with CVD death. These findings were showed when PP substituted for systolic BP in multivariate model. Additionally, in sensitivity analysis considering 


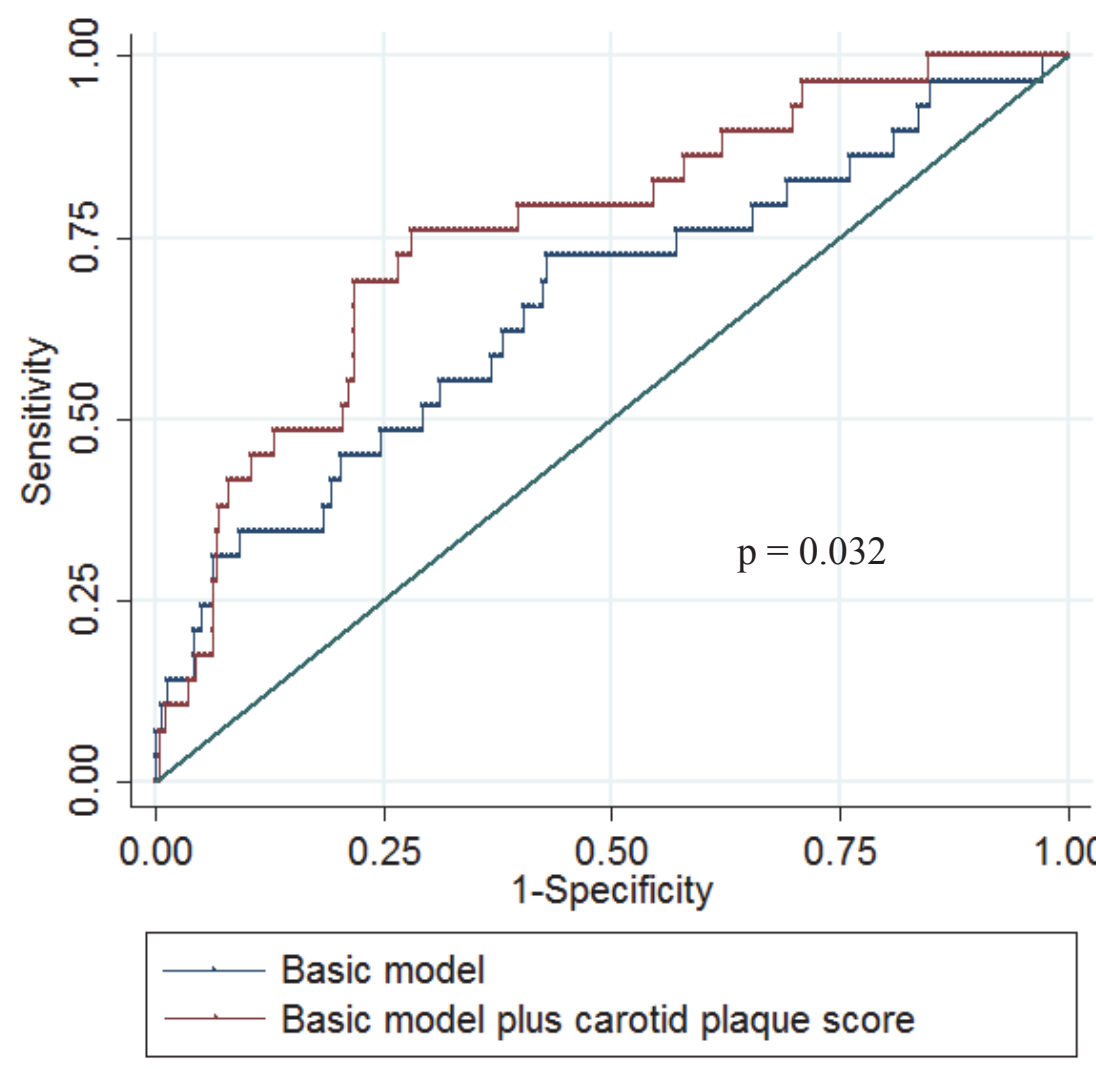

Fig. 2. Receiver operating characteristic curves showing the predictive values of two models for cardiovascular disease. The basic model included sex, age, body mass index, systolic blood pressure, total cholesterol, HDL cholesterol, hemoglobin A1c, smoking status, estimated GFR, C-reactive protein (log-transformed), and asymmetric dimethylarginine (log-transformed). The C statistic was 0.657 for the basic model and 0.758 for the basic model plus the carotid plaque score.

competing risk of death from any causes other than CVD, the SHR for the high CPS group was significantly higher than that for the no CPS group after adjusting for potential confounders (Table 3; SHR, 3.14; 95\% CI, 1.06-9.30) in the multivariate analysis.

To examine if ADMA moderated the association between CPS and CVD mortality, we performed the multivariate analysis stratified by median ADMA level. The significant association between the CPS and CVD death was observed in participants with lower ADMA level, but not in higher ADMA level (Table 4).

Predictive values for CVD obtained with the basic model and basic model plus CPS groups were compared in a ROC curve analysis. The results are shown in Fig. 2. The $\mathrm{C}$ statistics were 0.657 (95\% CI, $0.543-0.771)$ for the basic model and 0.758 (95\% CI, 0.666-0.851) for the basic model plus CPS groups. Compared to the basic model alone, the addition of the CPS groups significantly increased the discriminative power $(p=0.032)$.

\section{Discussion}

In a community-living oldest old cohort (age $\geq 85$ years) with no history of CVD, we first demonstrated a correlation between a high CPS and CVD death. Although previous studies have reported an association between CPS and CVD incidence or mortality in Japanese adults ${ }^{25-27)}$, the mean participant ages were lower in those studies. Participants selected for our cohort also had a low risk of CVD, characterized by a lack of CVD history, low prevalence of diabetes and dyslipidemia, and relatively low BMI. Nevertheless, the prevalence of carotid plaque was $61.1 \%$, and the 6-year mortality of CVD only increased among those with a high plaque burden. We, therefore, conclude that a lifelong atherosclerosis burden, assessed quantitatively using carotid plaque measurements, was associated with an increased risk of CVD even in a very elderly, extremely low-risk population.

In older populations, traditional CVD risk fac- 
tors (e.g., systolic BP, TC, and current smoking) have a relatively low predictive value for CVD or CVDrelated mortality relative to their efficacies in younger populations ${ }^{7,28)}$. Therefore, several studies have sought to identify markers that could reliably predict CVD or CVD-related mortality in older populations ${ }^{7,28-31)}$. In the present study, we investigated whether CPS would increase the discriminatory power for CVD prediction. Notably, the C statistic was 0.758 after adding CPS to a basic prediction model based on traditional risk factors; this value was higher than that of the basic model (0.657), indicating a significant impact of adding CPS to traditional CVD risk factors. A previous study of participants aged 45-84 years reported that the presence of carotid plaques independently predicted CVD and improved risk predictions for coronary heart disease events over a period of 7.8 years when added to the Framingham risk factors ${ }^{12)}$, and our results were consistent with the results of that study. We assumed that classical atherosclerosis risk factors would have little effect on CVD mortality in our cohort, possibly because at a late stage of life, these factors might not represent actual lifetime exposure to the same risk factors. Therefore, we speculate that an ultrasound examination of carotid plaque, a surrogate marker of the lifelong atherosclerotic burden, might facilitate CVD death risk evaluation even in elderly subjects with a very low cardiovascular risk.

By contrast, we were unable to demonstrate an association between ADMA and cardiovascular mortality in the present study. However, we will present some possible explanations for our results. The results of accumulating epidemiological and clinical studies of the association between ADMA concentrations and cardiovascular outcomes are conflicting and might have been modulated by population characteristics. In patients with pre-existing CVD, ADMA was associated with cardiovascular mortality or non-fatal myocardial infarction even after adjusting for traditional risk factors and B-type natriuretic peptide ${ }^{15}$. ADMA was associated with an increased risk of all-cause and cardiovascular deaths in patients with stage 3-4 chronic kidney disease ${ }^{16)}$. In contrast, the ADMA concentration was significantly associated with all-cause mortality, but not CVD incidence, in a general middle aged population ${ }^{18)}$ and in very elderly communitydwelling individuals ${ }^{32)}$. In our very old, entirely CVDfree participants, the ability of ADMA to predict allcause and cardiovascular mortality might be limited. Our multivariate analysis failed to find an association of ADMA with all-cause mortality during the 6-year follow-up (HR, 1.54; 95\% CI, 0.35-6.79). Second, ADMA induces endothelial dysfunction by inhibiting NO synthesis; accordingly, the availability of L-argi- nine, a NO precursor, could modify the effect of ADMA on cardiovascular outcomes. In an Italian cohort of the very old, ADMA predicted all-cause mortality among participants with L-arginine levels $<60 \mu \mathrm{M} / \mathrm{L}$, but did not predict among those with L-arginine levels $>60 \mu \mathrm{M} / \mathrm{L}^{32}$. Unfortunately, L-arginine levels were unavailable in our study; however, this notion should be tested in future.

Despite no associations between ADMA and cardiovascular mortality, ADMA moderated association between CPS and CVD death, in which CPS was significantly associated CVD death only in those with lower ADMA levels. Because of observational nature of this study, we couldn't address the issue precisely. However, those who had higher ADMA levels were older and had lower eGFR levels than their counter parts (data not shown), so older age and poor renal function might be confounded association between CPS and cardiovascular mortality.

The present study had several limitations. First, the carotid plaque definition differed from that used in other reports ${ }^{72}$. We defined a carotid plaque as a clearly identified area of focally increased thickness $(>1.2 \mathrm{~mm})$ in the intima-media layer to distinguish plaques clearly from carotid IMT. Previous studies demonstrated that carotid IMT at plaque-free sites in common carotid arteries increased with chronological age (up to 100 years) in a Japanese cohort ${ }^{23)}$, suggesting that IMT thickening might be a stage in physiological vascular aging in the oldest old. Furthermore, a recent large-scale study reported that three-dimensional ultrasound-assessed carotid plaque volume was associated with a greater carotid atherosclerosis burden than had previously been reported ${ }^{33)}$. Very recently, 18-Fluorodeoxyglucose (18F-FDG) positron emission tomography (PET) is a useful technique for detecting inflamed atherosclerotic plaques in vivo ${ }^{34)}$. Although we applied a simple two-dimensional ultrasound technique to determine this burden in vulnerable participants, the lack of precise carotid plaque quantification might have led to an underestimation. Second, we investigated only a limited number of biomarkers. As serum homocysteine levels have been reported to improve CVD risk predictions in the oldest old ${ }^{7}$, we propose that this parameter should be included in future studies. In addition, it might be appropriate to include aging-sensitive biomarkers such as leukocyte telomere length in future studies of CVD risk in the oldest old. Finally, our study might have been underpowered because of the relatively small sample size and relatively short follow-up period. In addition, we could not evaluate the impact of CPS on specific CVD death subtypes (e.g., coronary heart disease, ischemic stroke, or cerebral hemorrhage deaths) 
because of the small number of each type of event. We, therefore, recommend conducting a larger cohort study with a longer follow-up period to confirm our findings in the future.

\section{Conclusion}

A high CPS was significantly associated with an increase in cardiovascular mortality in our cohort of oldest old individuals with an extremely low CVD risk. ADMA was not associated with CVD death, but the significant association between CPS and CVD death was observed only in lower ADMA level. Despite the modest improvement in CVD predictability achieved by adding CPS measurements to traditional risk factors, our study provides epidemiological evidence that the lifelong atherosclerosis burden correlates with CVD risk in individuals aged $\geq 85$ years. Our results may have clinical relevance to the identification of patients who would be amenable to primary CVD prevention through lifestyle modification.

\section{Acknowledgments}

We thank the participants and their families for their time and personal information, and Ms. Miho Shimura for her kind assistance.

\section{Source of Funding}

The TOOTH study is funded by a Grant-in-Aid for Scientific Research (C) (No 20590706, 21590775) from the Japan Society for the Promotion of Science, the medical-welfare-food-agriculture collaborating consortium project from the Japan Ministry of Agriculture, Forestry, and Fisheries, a grant from the Novartis Foundation for Gerontological Research, a grant from the Foundation for Total Health Promotion, and the Chiyoda Mutual Life Foundation. The funding agencies had no direct role in the design or conduct of the study; the collection, management, analysis, or interpretation of the data; or the preparation or approval of the manuscript.

\section{Conflicts of Interest}

Arai received research grant funding from Daiichi Sankyo Company, Limited. All other authors have nothing to disclose.

\section{References}

1) Rosamond W, Flegal K, Friday G, Furie K, Go A, Greenlund K, Haase N, Ho M, Howard V, Kissela B, Kittner S,
Lloyd-Jones D, McDermott M, Meigs J, Moy C, Nichol G, O’Donnell CJ, Roger V, Rumsfeld J, Sortie P, Steinberger J, Thom T, Wasserthiel-Smoller S, and Hong Y: American Heart Association Statistics Committee and Stroke Statistics Subcommittee: Heart disease and stroke statistics - 2007 update: a report from the American Heart Association statistics committee and stroke statistics subcommittee. Circulation, 2007; 115: e69-e171

2) Dinç G, Sözmen K, Gerçeklioğlu G, Arık H, Critchley J, and Unal B: Decreasing trends in cardiovascular mortality in Turkey between 1988 and 2008. BMC Public Health, 2013; 13: 896

3) Zhou T, Li X, Tang Z, Xie C, Tao L, Pan L, Huo D, Sun F, Luo Y, Wang W, Yan A and Guo X: Risk factors of CVD mortality among the elderly in Beijing, 1992-2009: an 18-year cohort study. Int J Environ Res Public Health, 2014; 11: 2193-2208

4) Goff DC Jr, Lloyd-Jones DM, Bennett G, Coady S, D’Agostino RB, Gibbons R, Greenland P, Lackland DT, Levy D, O’Donnell CJ, Robinson JG, Schwartz JS, Shero ST, Smith SC Jr, Sorlie P, Stone NJ, Wilson PW, Jordan HS, Nevo L, Wnek J, Anderson JL, Halperin JL, Albert NM, Bozkurt B, Brindis RG, Curtis LH, DeMets D, Hochman JS, Kovacs RJ, Ohman EM, Pressler SJ, Sellke FW, Shen WK, Smith SC Jr, and Tomaselli GF: American College of Cardiology/American Heart Association Task Force on Practice Guidelines: 2013 ACC/AHA guideline on the assessment of cardiovascular risk: a report of the American College of Cardiology/American Heart Association Task Force on Practice Guidelines. Circulation, 2014; 129: S49-S73

5) Wilson PW, D'Agostino RB, Levy D, Belanger AM, Silbershatz H, and Kannel WB: Prediction of coronary heart disease using risk factor categories. Circulation, 1998; 97: 1837-1847

6) Hippisley-Cox J, Coupland C, Vinogradova Y, Robson J, Minhas R, Sheikh A, and Brindle P: Predicting cardiovascular risk in England and Wales: prospective derivation and validation of QRISK2. BMJ, 2008; 336: 1475-1482

7) de Ruijter W, Westerndorp RG, Assendelft WJ, den Elzen WP, de Craen AJ, le Cessie S, and Gussekloo J: Use of Framingham risk score and new biomarkers to predict cardiovascular mortality in older people: population based observational cohort study. BMJ, 2009; 338: a3083

8) Kannel WB: Coronary heart disease risk factors in the elderly. Am J Geriatr Cardiol, 2002; 11: 101-107

9) Murakami K, Asayama K, Satoh M, Inoue R, TsubotaUtsugi M, Hosaka M, Matsuda A, Nomura K, Murakami T, Kikuya M, Metoki H, Imai Y, and Ohkubo T: Risk Factors for Stroke among Young-Old and Old-Old Community-Dwelling Adults in Japan: the Ohasama Study. J Atheroscler Thromb, 2017; 24: 290-300

10) Negi SI and Nambi V: The role of carotid intimal thickness and plaque imaging in risk stratification for coronary heart disease. Curr Atheroscler Rep, 2012; 14: 115-123

11) Nambi V, Chambless L, Folsom AR, He M, Hu Y, Mosley T, Volcik K, Boerwinkle E, and Ballantyne CM: Carotid intima-media thickness and presence or absence of plaque improves prediction of coronary heart disease risk: the ARIC (Atherosclerosis Risk In Communities) study. J Am Coll Cardiol, 2010; 55: 1600-1607 
12) Polak JF, Szkio M, Kronmal RA, Burke GL, Shea S, Zavodni AE, and O'Leary DH: The value of carotid artery plaque and intima-media thickness for incident cardiovascular disease: the multi-ethnic study of atherosclerosis. J Am Heart Assoc, 2013; 2: e000087

13) Plichart M, Celermajer DS, Zureik M, Helmer C, Jouven $\mathrm{X}$, Ritchie K, Tzourio C, Ducimetière P, and Empana JP: Carotid intima-media thickness in plaque-free site, carotid plaques and coronary heart disease risk prediction in older adults. The Three-City Study. Atherosclerosis, 2011; 219: 917-924

14) Krempl TK, Maas R, Sydow K, Meinertz T, Böger RH, and Kähler J: Elevation of asymmetric dimethylarginine in patients with unstable angina and recurrent cardiovascular events. Eur Heart J, 2005; 26: 1846-1851

15) Schnabel R, Blankenberg S, Lubos E, Lackner KJ, Rupprecht HJ, Espinola-Klein C, Jachmann N, Post F, Peetz D, Bickel C, Cambien F, Tiret L, and Münzel T: Asymmetric dimethylarginine and the risk of cardiovascular events and death in patients with coronary artery disease: results from the AtheroGene Study. Circ Res, 2005; 97: e53-e59

16) Young JM, Terrin N, Wang X, Greene T, Beck GJ, Kusek JW, Collins AJ, Sarmak MJ, and Menon V: Asymmetric dimethylarginine and mortality in stages 3 to 4 chronic kidney disease. Clin J Am Soc Nephrol, 2009; 4: 11151120

17) Lajer M, Tamow L, Jorsal A, Teerlink T, Parving HH, and Rossing P: Plasma concentration of asymmetric dimethylarginine (ADMA) predicts cardiovascular morbidity and mortality in type 1 diabetic patients with diabetic nephropathy. Diabetes Care, 2008; 31: 747-752

18) Böger RH, Sullivan LM, Schwedhelm E, Wang TJ, Maas R, Benjamin EJ, Schulze F, Xanthakis V, Benndorf RA, and Vasan RS: Plasma asymmetric dimethylarginine and incidence of cardiovascular disease and death in the community. Circulation, 2009; 119: 1592-1600

19) Bode-Böger SM, Scalera F, and Martens-Lobenhoffer J: Asymmetric dimethylarginine (ADMA) accelerates cell senescence. Vasc Med, 2005; 10: S65-S71

20) Arai $Y$, Iinuma $T$, Takayama $M$, Takayama $M$, Abe $Y$, Fukuda R, Ando J, Ohta K, Hanabusa H, Asakura K, Nishiwaki Y, Gondo Y, Akiyama H, Komiyama K, Gionhaku N, and Hirose N: The Tokyo Oldest Old Survey on Total Health (TOOTH): a longitudinal cohort study of multidimensional components of health and well-being. BMC Geriatr, 2010; 10: 35

21) Matsuo S, Imai E, Horio M, Yasuda Y, Tomita K, Nitta K, Yamagata K, Tomino Y, Yokoyama H, and Hishida A; Collaborators developing the Japanese equation for estimated GFR: Revised equations for estimated GFR from serum creatinine in Japan. Am J Kidney Dis, 2009; 53: 982-992

22) O'Leary DH, Polak JF, Kronmal RA, Manolio TA, Burke GL, and Wolfson SK Jr: Carotid-artery intima and media thickness as a risk factor for myocardial infarction and stroke in older adults. Cardiovascular Health Study Collaborative Research Group. N Engl J Med, 1999; 340: $14-22$

23) Homma $S$, Hirose N, Ishida $H$, Ishii $T$, and Araki G: Carotid plaque and intima-media thickness assessed by b-mode ultrasonography in subjects ranging from young adults to centenarians. Stroke, 2001; 32: 830-835

24) Fine JP and Gray RJ: A proportional hazards model for the subdistribution of a competing risk. J Am Stat Assoc 1999; 94: 496-509

25) Kadota A, Miura K, Okamura T, Fujiyoshi A, Ohkubo T, Kadowaki T, Takashima N, Hisamatsu T, Nakamura Y, Kasagi F, Maegawa H, Kashiwagi A, and Ueshima $\mathrm{H}$ : SESSA Research Group; NIPPON DATA80/90 Research Group: Carotid intima-media thickness and plaque in apparently healthy Japanese individuals with an estimated 10-year absolute risk of CAD death according to the Japan Atherosclerosis Society (JAS) guidelines 2012: the Shiga Epidemiological Study of Subclinical Atherosclerosis (SESSA). J Atheroscler Thromb, 2013; 20: 755-766

26) Kitamura $A$, Iso $H$, Imano $H$, Ohira $T$, Okada $T$, Sato $S$, Kiyama M, Tanigawa T, Yamagishi K, and Shimamoto T: Carotid intima-media thickness and plaque characteristics as a risk factor for stroke in Japanese elderly men. Stroke, 2004; 35: 2788-2794

27) Handa N, Matsumoto M, Maeda H, Hougaku $H$, and Kamada T: Ischemic stroke events and carotid atherosclerosis. Results of the Osaka Follow-up Study for Ultrasonographic Assessment of Carotid Atherosclerosis (the OSACA Study). Stroke, 1995; 26: 1781-1786

28) Odden MC, Shlipak MG, Whitson HE, Katz R, Kearney PM, Defilippi C, Shastri S, Sarnak MJ, Siscovick DS, Cushman M, Psaty BM, and Newman AB: Risk factors for cardiovascular disease across the spectrum of older age: the Cardiovascular Health Study. Atherosclerosis, 2014; 237: 336-342

29) Cao JJ, Biggs ML, Barzilay J, Konen J, Psaty BM, Kuller L, Bleyer AJ, Olson J, Wexler J, Summerson J, and Cushman M: Cardiovascular and mortality risk prediction and stratification using urinary albumin excretion in older adults ages 68-102: the Cardiovascular Health Study. Atherosclerosis, 2008; 197: 806-813

30) Dutta A, Henley W, Pilling LC, Wallace RB, and Melzer D: Uric acid measurement improves prediction of cardiovascular mortality in later life. J Am Geriatr Soc, 2013; 61:319-326

31) Wannamethee SG, Sattar N, Papcosta O, Lennon L, and Whincup PH: Alkaline phosphatase, serum phosphate, and incident cardiovascular disease and total mortality in older men. Arterioscler Thromb Vasc Biol, 2013; 33: 1070-1076

32) Pizzarelli F, Maas R, Dattolo P, Tripepi G, Michelassi S, D’Arrigo G, Mieth M, Bandinelli S, Ferrucci L, and Zoccali C: Asymmetric dimethylarginine predicts survival in the elderly. Age (Dordr), 2013; 35: 2465-2475

33) Sillesen H, Muntendam P, Adourian A, Entrekin R, Garcia M, Falk E, and Fuster V: Carotid plaque burden as a measure of subclinical atherosclerosis: comparison with other tests for subclinical arterial disease in the High Risk Plaque Bioimage study. JACC Cardiovasc Imaging, 2012; 5: 681-689

34) Poredos P, Spirkoska A, Lezaic L, Mijovski MB, and Jezovnik MK: Patients with an inflamed atherosclerotic plaque have increased levels of circulating inflammatory markers. J Atheroscler Thromb, 2017; 24: 39-46 